\title{
EXPERIMENTAL VALIDATION OF A CONTROL METHOD FOR UNDERACTUATED BIPEDAL WALKING ON COMPLIANT GROUND
}

\author{
Daojin Yao, ${ }^{*}$ Yao Wu, ${ }^{*}$ Yang Wang, ${ }^{*}$ and Xiaohui Xiao*
}

\begin{abstract}
This paper presents the experimental validation of an adaptive feedforward control (AFC) method for underactuated bipedal walking on compliant ground using UBbot, a four-link planar robot prototype composed of two legs with knees and unactuated point feet. In previous studies, compliant ground was modelled as a springdamper system, and stable walking was achieved by adjusting the horizontal velocity of the biped's centre of mass (CoM) to achieve a suggested value that has been proved available for realizing at least one walking cycle. This study introduces a control scheme to implement AFC in UBbot. First, the robot CoM position in the next cycle is calculated based on the CoM horizontal velocity variation in the current cycle using AFC. Then, the corresponding gait is obtained by the robot CoM position by using the method of virtual constraints. Finally, based on feedback linearization, underactuated bipedal walking is realized through torque control. Walking experiments were performed on ground made of three types of materials: concrete, particle board, and rubber. The results showed that AFC effectively suppressed the attenuation of walking speed caused by ground compliance, and dynamic walking with a step of $31 \%$ of the leg length was successfully realized on compliant ground.
\end{abstract}

\section{Key Words}

Experimental validation, underactuated bipedal walking, compliant ground

\section{Nomenclature}

$w v$ robot walking speed

$x_{\mathrm{f}}$ horizontal displacement of CoM

$x_{\mathrm{fc}}$ horizontal displacement of CoM for control cycle

$u_{\mathrm{f}}$ horizontal velocity of $\mathrm{CoM}$

* School of Power and Mechanical Engineering, Wuhan University, Wuhan 430072, China; e-mail: \{ydaojin, wuyao, wangyang.hf1987, xhxiao\}@whu.edu.cn

Corresponding author: Xiaohui Xiao

Recommended by Prof. Enver Tatlicioglu

(DOI: 10.2316/Journal.206.2018.5.206-5137)

\author{
$u_{\mathrm{fd}} \quad$ ideal horizontal velocity of CoM \\ $u_{\mathrm{fc}}$ horizontal velocity of CoM for control cycle \\ $\boldsymbol{q}$ vector of robot's joints position \\ $\dot{q} \quad$ vector of robot's joints velocity \\ $\ddot{q} \quad$ vector of robot's joints accelerated velocity \\ $\boldsymbol{q}_{\mathrm{fc}} \quad$ vector of robot position at the terminal time of \\ SSP with the AFC \\ $\boldsymbol{q}_{a} \quad$ vector of actuated coordinates \\ $\boldsymbol{e} \quad$ vector of the virtual constraints error \\ $j \quad$ number of walking cycle \\ $p \quad$ sampling points during a walking cycle \\ $N \quad$ total number of sampling points during a \\ walking cycle
}

\section{Introduction}

Underactuated bipedal walking has attracted increasing attention in recent years [1]. Several well-known underactuated bipedal robots such as RABBIT [2], MABEL [3], and AMBER2 [4] have been designed and proposed. In most previous studies, a rigid walking surface has been assumed, and ground compliance has not been considered [5], [6]. However, the ground is often made of compliant materials such as plastic, wood, and rubber; furthermore, robots' mass and power consumption have been increasing [7]. A realistic walking surface should ideally be compliant, and ground compliance is no longer a negligible effect to realize stable and efficient underactuated bipedal walking in a real environment [8].

Many studies have demonstrated the effect of ground compliance on underactuated bipedal walking [9], [10]. Experiments with the RABBIT prototype have revealed that the walking speed on a compliant surface was lower than that on a rigid one, mainly owing to additional energy loss during compliant impact [9]. Aguilar used hydrostatic and hydrodynamic methods to study the effects of nonlinear friction and hydrodynamic resistance on sandy soil particles during the deformation of foot plant and sandy 
ground, and walking control on compliant ground was discussed from the viewpoint of mechanics [10]. However, modelling the exact contact model between the robot and the compliant ground is difficult [9].

Several methodologies have been considered to improve the walking performance of a bipedal robot walking on compliant ground. In [11], a compliant contact model for a real environment was built through numerical calculations; one periodic stable gait based on the mentioned model was designed, and subsequently, a PD feedback controller for stable walking and velocity regulation was implemented. In [12], an artificial neural network method was applied to identify the mapping relationship between Runbot's walking speed and each joint state, and then, the walking stability was improved by regulating the walking speed by controlling each joint motion. Although the aforementioned method achieves stable walking on compliant ground, limited by the identification of the robot structure and the actual ground environment, these methods are essentially non-general for all underactuated bipedal walking systems. In previous studies, an AFC method was proposed and validated through simulations to stabilize underactuated bipedal walking on compliant ground [13].

The main contribution of this study is to validate AFC using a four-link planar bipedal robot called UBbot that has two legs with knees and unactuated point feet. A walking control scheme is proposed to implement AFC on UBbot. In the control scheme, UBbot's CoM position in the next cycle is calculated using AFC by regulating the CoM horizontal velocity. Then, the walking gait is obtained from the robot's CoM position by using the method of virtual constraints. Finally, stable walking is realized by implementing feedback linearization. In this paper, a more direct and simpler stability criterion based on walking speed is proposed. According to walking experiments on ground made of three types of materials - concrete, particle board, and rubber - the effectiveness of the control strategy and availability of the experimental scheme were proved.

The remainder of this paper is organized as follows. Section 2 summarizes the AFC method. Section 3 proposes a control scheme for implementing $\mathrm{AFC}$ in a real robot prototype. Section 4 describes the configuration of UBbot and experimental validation of AFC on compliant ground. Finally, Section 5 presents the conclusion of this study.

\section{AFC for Underactuated Bipedal Walking on Compliant Ground}

\subsection{Compliant Ground Equivalence}

Compliant ground is modelled as a spring-damper equivalent system [13], which is constructed with a group of independent compliant structures, as shown in Fig. 1(a). A compliant structure comprises a discrete elastic spring, viscous damp cell arrays, rigid ground foundation, and surface fragments. The walking process of a four-link robot on compliant ground is shown in Fig. 1(b). Based on the contact status between the leg end and the ground, one walking cycle could be divided into two successive phases: a single support phase (SSP) and a double support phase

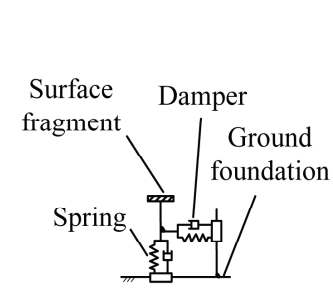

(a)

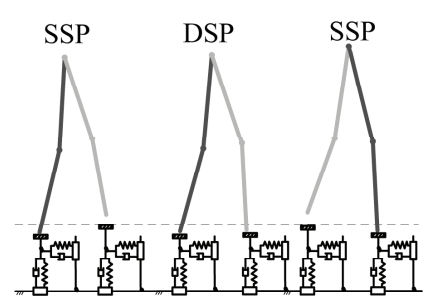

(b)
Figure 1. Walking on compliant ground.

(DSP). During the SSP, the leg standing on the ground is called the support foot and the other leg is called the swing foot.

\subsection{Definition of Stable Bipedal Walking}

The most intuitive definition of stable biped walking is likely that "the biped does not fall". This definition is very simple and intuitive; however, it is difficult to rigorously verify the stability of a given controller according to this definition. A new definition of underactuated bipedal stable walking is proposed as follows: if the robot walking speed (defined as step length/period of a full walking cycle) can always converge to a certain range that has been proved available for realizing at least one walking cycle, the robot walking is stable.

Definition 1 (Fall). Let $Q_{\text {Fall }}$ be the state space in which the robot walking speed is beyond the range of stable walking speed during a walking cycle. If a robot state $x \in Q_{\text {Fall }}$, the robot is falling.

Based on Definition 1, falling is the state in which the robot enters the fall state set. A biped robot is stable if and only if it can always control itself and avoid entering the fall state set. The fall state set $Q_{\text {Fall }}$ can be represented as:

$Q_{\text {Fall }}=\left\{\dot{\boldsymbol{x}} \mid w v(\dot{\boldsymbol{x}})=<w v_{\text {min }}(\dot{\boldsymbol{x}})\right\} \cup\left\{\dot{\boldsymbol{x}} \mid w v(\dot{\boldsymbol{x}})>=w v_{\max }(\dot{\boldsymbol{x}})\right\}$

where $w v(\dot{\boldsymbol{x}})$ is the biped walking speed, and $w v_{\max }(\dot{\boldsymbol{x}})$ and $w v_{\min }(\dot{\boldsymbol{x}})$ are the maximum and minimum values that have been proved available for realizing at least one walking cycle, respectively.

Definition 2 (Basin of Fall). Let $B \in \mathbb{R}^{N}$ be a subset of the biped walking state space. $\forall x \in B \Rightarrow \exists \Delta t \geq 0$ s.t. $x(t+\Delta t) \in Q_{\text {Fall }}$, where $B$ is called the basin of fall.

Definition 3 (Stable Walking). Biped walking is stable if the state space of walking motion does not intersect with $\mathrm{B}$.

\subsection{Review of Control Strategy}

Inspired by the potential use of humanoid robots in real environments, an AFC strategy is proposed to stabilize 


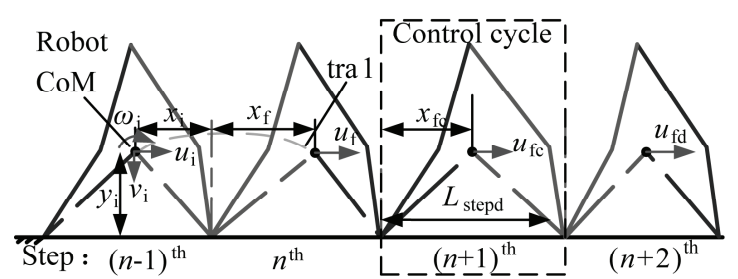

(a)

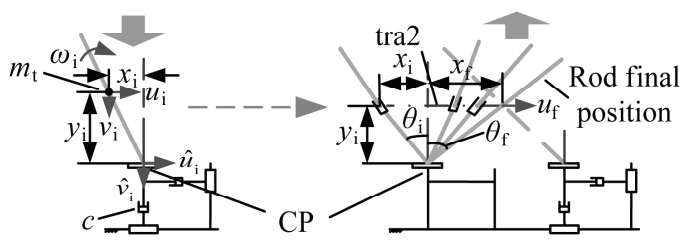

(b)

(c)

Figure 2. Schematic of AFC.

underactuated bipedal walking on compliant ground. By observing humans' gait, humans' velocity could be regulated by adjusting their posture: when the body leans forward, the walking speed increases, and when the body leans back, the walking speed decreases automatically. Based on these principles, an AFC method based only on this state of the robot's CoM is proposed.

In the control system, $u_{\mathrm{f}}$ is the output, and $x_{\mathrm{f}}$ is the input. Based on the state at the current cycle, $x_{\mathrm{f}}$ is calculated to let $u_{\mathrm{f}}$ track $u_{\mathrm{fd}}$ for the next cycle, and thus, stable walking is realized. To identify the relationship between $u_{\mathrm{f}}$ and $x_{\mathrm{f}}$, an equivalent rod-ground coupling model is established, as shown in Fig. 2. Corresponding to both phases of a full walking cycle, two sub-models impact and swing - are constructed in the sequence.

In the impact sub-model, the final states of the rod's CoM under are given as [13]:

$$
\left\{\begin{array}{l}
u=u\left(u_{i}, \lambda, \rho, y_{i}, \hat{u}_{i}\right) \\
v=v\left(v_{i}, \lambda, \rho, x_{i}, y_{i}, \hat{v}_{i}, \hat{u}_{i}\right) \\
\omega=\omega\left(\omega_{i}, \lambda, \rho, x_{i}, y_{i}, \hat{v}_{i}, \hat{u}_{i}\right)
\end{array}\right.
$$

where $u$ and $v$ denote, respectively, the horizontal and vertical velocities of the $\mathrm{CoM} ; \omega$ denotes the rotational angular velocity of the CoM; $x$ and $y$ denote, respectively, the horizontal and vertical displacements of the CoM to the rod-ground contact point $(\mathrm{CP})$ at the initial time of impact; and $\rho$ denotes the gyration radius of the rod. The subscripts "i", " $\wedge$ ", and " $\sim$ " denote the initial value, state at the $\mathrm{CP}$, and state under only the horizontal ground reaction force, respectively. $\lambda$ is the attenuation coefficient of the CoM horizontal velocity relative to the CP.

In the swing sub-model, a straight-line trajectory is designed for the inverted pendulum's CoM trajectory as shown in Fig. 2(c). The total variation of the kinetic energy $\Delta W$ is given as:

$$
\Delta W=(1-\lambda) \int_{\theta_{\mathrm{i}}}^{\theta_{\mathrm{f}}} F d r=(1-\lambda) m_{\mathrm{tot}} g\left(x_{\mathrm{f}}^{2}-x_{\mathrm{i}}^{2}\right) /\left(2 y_{\mathrm{i}}\right)
$$

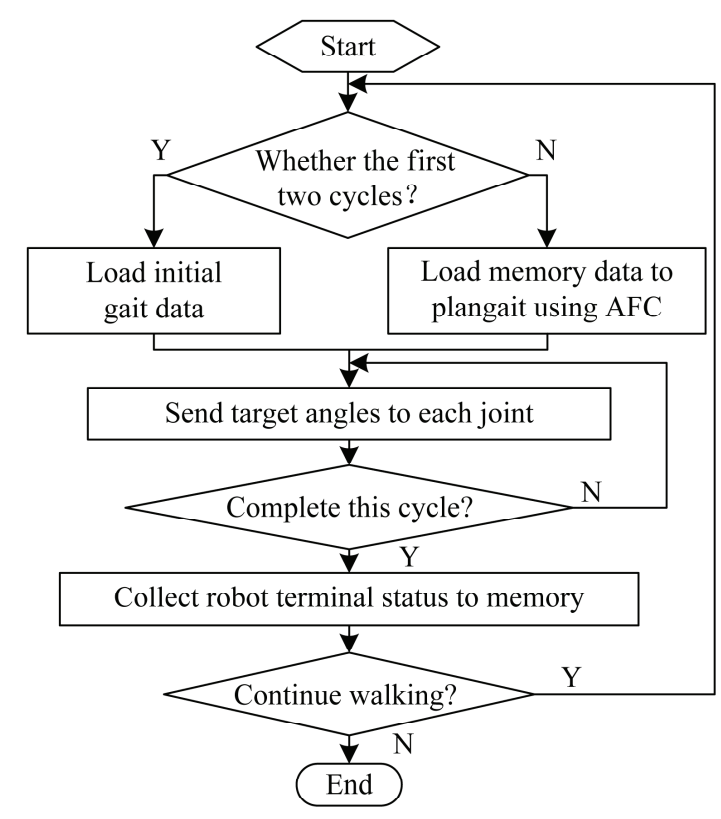

Figure 3. Flowchart of control system.

where $F$ denotes the contact force from the ground to the rod; $m_{\text {tot }}$ is the robot's total mass; $g$ denotes the acceleration due to gravity; $\theta$ denotes the angle of the swing phase; and the subscripts "i" and "f" denote the initial and final states, respectively.

Therefore, $u_{\mathrm{fc}}$ is obtained as follows:

$$
u_{\mathrm{fc}}=\boldsymbol{U}\left(\lambda, v_{\mathrm{i}}, u_{\mathrm{i}}, x_{\mathrm{i}}, x_{\mathrm{f}}, y_{\mathrm{i}}\right)
$$

The CoM displacement $x_{\mathrm{fc}}$ could be obtained by linearization at $x=x_{\mathrm{i}}$ as follows:

$$
x_{\mathrm{fc}}=x_{\mathrm{f}}+\frac{u_{\mathrm{fd}}-\boldsymbol{U}\left(\lambda, v_{\mathrm{f}}, u_{\mathrm{f}}, x_{\mathrm{i}}, L_{\text {stepd }}-x_{\mathrm{i}}, y_{\mathrm{f}}\right)}{\left.\frac{\partial}{\partial x} \boldsymbol{U}\left(\lambda, v_{\mathrm{f}}, u_{\mathrm{f}}, x_{\mathrm{i}}, L_{\text {stepd }}-x_{\mathrm{i}}, y_{\mathrm{f}}\right)\right|_{x=x_{\mathrm{i}}}}
$$

\section{AFC Implementation}

\subsection{Scheme for AFC Implementation}

Figure 3 presents the flowchart of the control system for implementing AFC for realizing stable walking with a real robot prototype. In the first two cycles, the robot tracks the pre-programmed initial gait. From the third cycle, the robot adjusts the gait online using AFC to achieve stable walking.

Figure 4 illustrates the detailed steps of the AFC working process in one cycle shown in Fig. 3. $\boldsymbol{q}_{r}, \dot{\boldsymbol{q}}_{r}$, $\boldsymbol{q}_{c}$, and $\dot{\boldsymbol{q}}_{c}$ denote the vector for the practical position, practical velocity, ideal position, and ideal velocity of the robot's joints, respectively. First, $u_{\mathrm{f}}$ in the $j-1$ cycle is calculated by the termination state of the robot. Then, according to $u_{\mathrm{f}}$, the AFC process adjusts $x_{\mathrm{fc}}$ of the $j$ cycle online to make $u_{\mathrm{f}}$ track $u_{\mathrm{fd}}$. Finally, the robot gait is planned using $x_{\mathrm{fc}}$, and the joint torque is calculated using the planned gait to control robot walking. In addition, the final state of the robot at the current cycle is recorded for calculating the next cycle. 


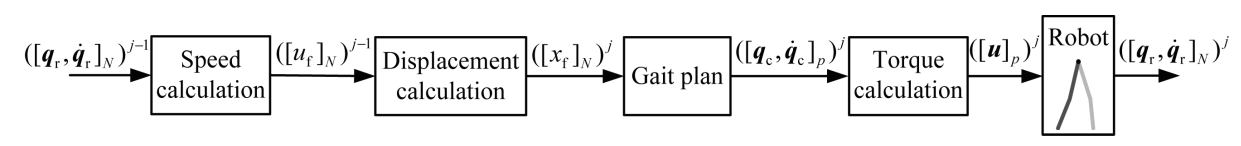

Figure 4. Block diagram of AFC working process in one cycle.

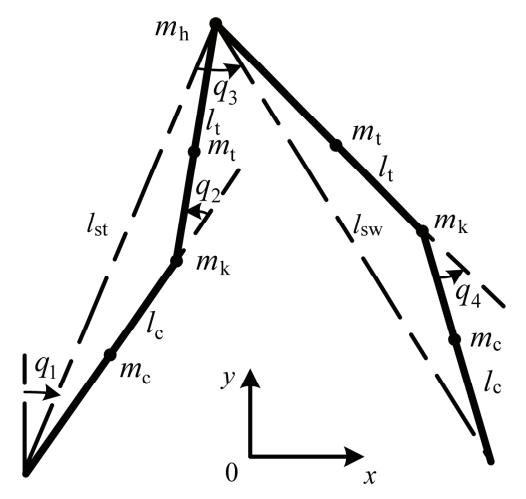

Figure 5. Definition of generalized coordinates and mechanical data of biped robot.

\subsection{Gait Plan}

Figure 5 shows the definition of the generalized coordinates and mechanical data for the four-link biped robot. The robot is composed of hips $\left(q_{3}\right)$ and two knees $\left(q_{2}, q_{4}\right)$ but no ankle. The lengths of the thigh and calf are $l_{t}$ and $l_{c}$, respectively. The masses of the thigh, calf, knee joint, and hip joint are $m_{t}, m_{c}, m_{k}$, and $m_{h}$, respectively. According to Routhian reduction, a variable to replace the time scale, the angle between the vertical line and the virtual leg $l_{s t}$ is defined as $q_{1}$. There is no actuated motor in $q_{1}$, and the model for the SSP is an underactuated mechanical system.

After determining the CoM displacement $\left(x_{\mathrm{f}}\right)$, robot's posture $\boldsymbol{q}_{\mathrm{fc}}=\left[q_{1 \mathrm{fc}}, q_{2 \mathrm{fc}}, q_{3 \mathrm{fc}}, q_{4 \mathrm{fc}}\right]^{\mathrm{T}}$ in the next cycle can be obtained by using inverse kinematics [14]. To realize UBbot walking, the method of virtual constraints is used here; this method has been proved successful in designing a feedback controller for stable walking of planar bipeds. Holonomic constraints are imposed on the robot's configuration by feedback control. Based on [13], the walking pattern can be given by the following holonomic constraints:

$$
\boldsymbol{e}=\boldsymbol{q}_{a}-\boldsymbol{h}_{\mathrm{d}}\left(q_{1}\right)
$$

where $\boldsymbol{e}$ is the $(3 \times 1)$ error vector between the actual value and the expected virtual constraints, $\boldsymbol{q}_{a}=\left[q_{2}, q_{3}, q_{4}\right]^{\mathrm{T}}$ is the vector of actuated coordinates, $q_{1}$ is a cyclic variable strictly increasing over a step to replace the time scale, and $\boldsymbol{h}_{\mathrm{d}}\left(q_{1}\right)$ is the $(3 \times 1)$ actuated variable vector as a function of $q_{1}$. Based on the robot's real initial states, $\left[\boldsymbol{q}_{\mathrm{i}}, \dot{\boldsymbol{q}}_{\mathrm{i}}\right]=\left[q_{1 \mathrm{i}}, q_{2 \mathrm{i}}, q_{3 \mathrm{i}}, q_{4 \mathrm{i}}, \dot{q}_{1 \mathrm{i}}, \dot{q}_{2 \mathrm{i}}, \dot{q}_{3 \mathrm{i}}, \dot{q}_{4 \mathrm{i}}\right]$, and the final state obtained by AFC, $\left[\boldsymbol{q}_{\mathrm{fc}}, \dot{\boldsymbol{q}}_{\mathrm{fc}}\right]=\left[q_{1 \mathrm{fc}}, q_{2 \mathrm{fc}}, q_{3 \mathrm{fc}}, q_{4 \mathrm{fc}}, \dot{q}_{1 \mathrm{f}}, \dot{q}_{2 \mathrm{f}}\right.$, $\left.\dot{q}_{3 \mathrm{f}}, \dot{\mathrm{q}}_{4 \mathrm{f}}\right]$, the actuated joint trajectory could be produced by Bézier polynomials of degree 3 .

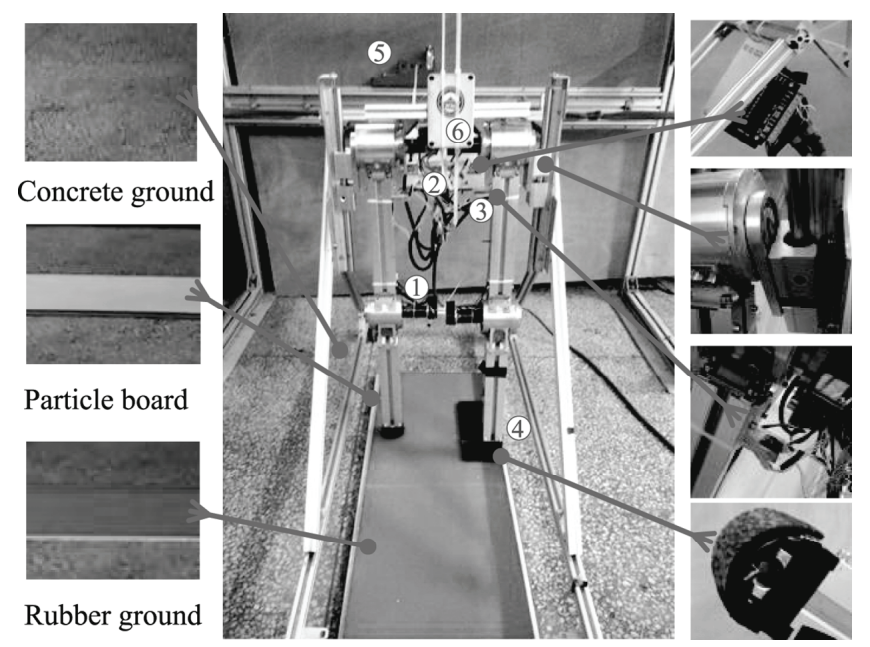

Figure 6. UBbot prototype. (1) Maxon DC motor RE25118752, (2) Servo amplifier RMDS102, (3) Gyroscope JY901, (4) Pressure sensor FSR406, (5) NI cRIO 9076, and (6) Arduino UNO.

Table 1

Parameters of UBbot

\begin{tabular}{|l|c|c|c|}
\hline$m_{\mathrm{c}}, m_{\mathrm{t}}(\mathrm{kg})$ & $m_{\mathrm{k}}(\mathrm{kg})$ & $m_{\mathrm{h}}(\mathrm{kg})$ & $l_{\mathrm{c}}, l_{\mathrm{t}}(\mathrm{m})$ \\
\hline 0.404 & 0.785 & 2.537 & 0.3 \\
\hline
\end{tabular}

\section{Experiments}

\subsection{Experimental Setup}

To validate AFC experimentally, a prototype of the UBbot planar bipedal robot with four links (two calves and two thighs, as shown in Fig. 6) was designed. This robot's four links are connected by revolute joints to form two symmetric legs. Actuators supply torque to each of the internal joints: one each at the knee and hip. Two drivers together move the hip joint. The contact between the half-cylindrical foot and the ground is assumed to be a line contact. To prevent motion in the frontal plane, the robot's hip joint contacts with the unpowered trolley via a bearing-sliding block-guideway.

UBbot has a height of $600 \mathrm{~mm}$ and total mass of $5.723 \mathrm{~kg}$. The mass of the unpowered trolley is $5.8 \mathrm{~kg}$. Table 1 lists the robot parameters.

For a real-time control platform, UBbot uses the NI cRIO9076 as the main controller. The pressure sensor FSR406 data can be recorded by the main controller by using the analogue signal acquisition card 9205 . Gyroscope data can be collected using the digital signal acquisition card 9401 to calculate the robot's spatial posture. 


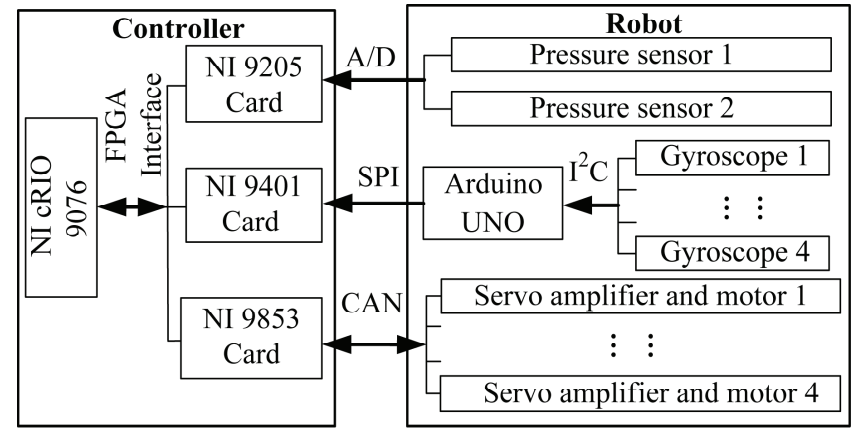

Figure 7. Structure of robot control system.

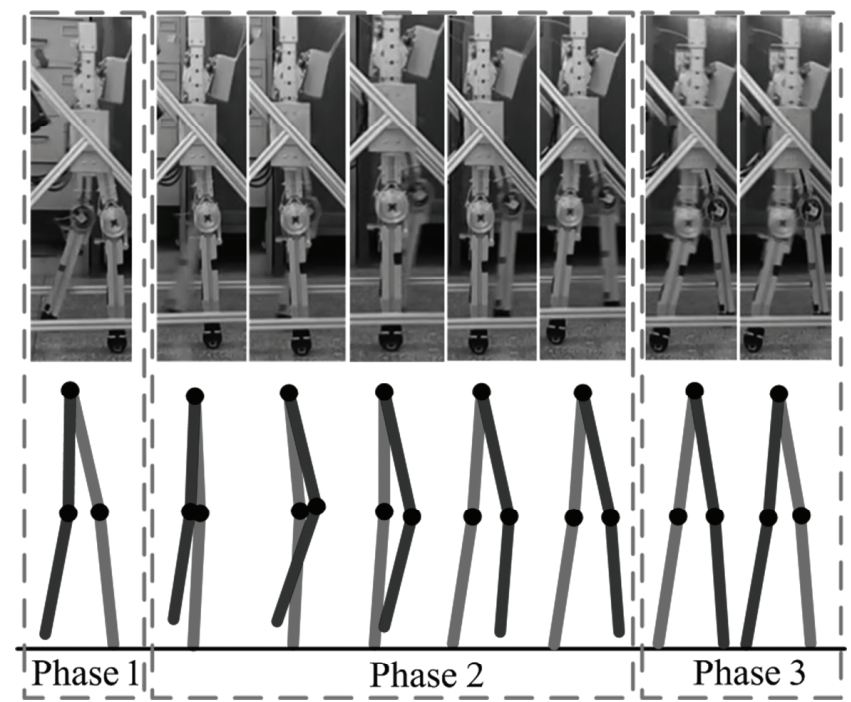

Figure 8. Video frames of a complete walking cycle of UBbot on concrete ground.

Robot joint movements can be controlled using the CAN communication card 9853 (Fig. 7). Owing to the sampling time of the gyroscope and response time of the servo amplifier, the sampling period of the real-time control system running is $7 \mathrm{~ms}(143 \mathrm{~Hz})$.

A set of asymptotically stable walking gaits with a step length of $0.1839 \mathrm{~m}$ under rigid robot-ground contact is calculated using MATLAB as $q_{\mathrm{fd}}=[0.2636,0.2500,0.2594$, $-0.1500]^{\mathrm{T}}, \dot{q}_{\mathrm{fd}}=[0.7425,-0.00026,-0.3593,1.3116]^{\mathrm{T}}$.

To validate the effectiveness of AFC, experiments were conducted with three different types of ground surfaces: concrete, particle board, and rubber; the particle board and rubber sheet were paved on the concrete surface. The structural parameters were as follows: the length and thickness of the particle board were 130 and $10 \mathrm{~mm}$, respectively, and the length and thickness of the rubber sheet were 130 and $5 \mathrm{~mm}$, respectively. To verify the adaptability of AFC, the particle board and rubber were alternately laid on the concrete ground, and a "rubberparticle board" ground surface was created.

\subsection{Walking without AFC}

Figure 8 shows a complete walking cycle of UBbot on concrete ground without AFC [15]. Figure 9 shows actual

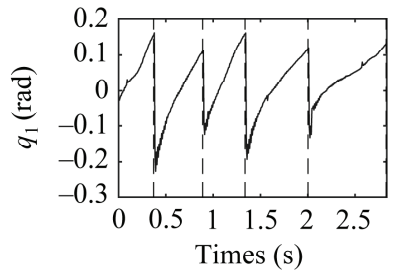

(a)

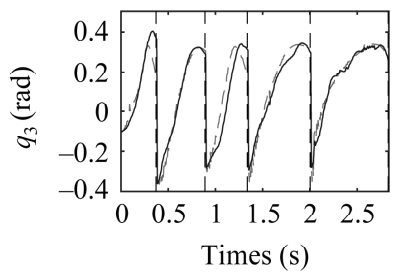

(c)

-- Desired values

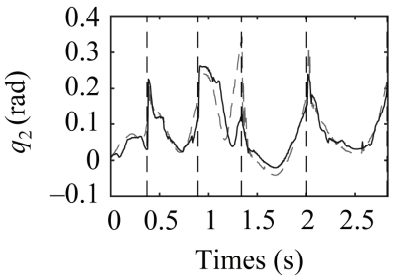

(b)

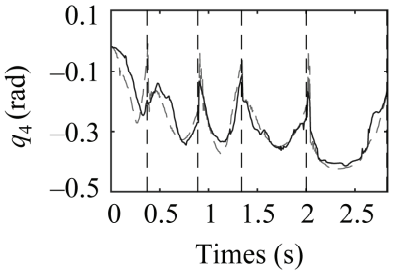

(d)

Real values
Figure 9. Actual trajectory tracking of virtual constraints.

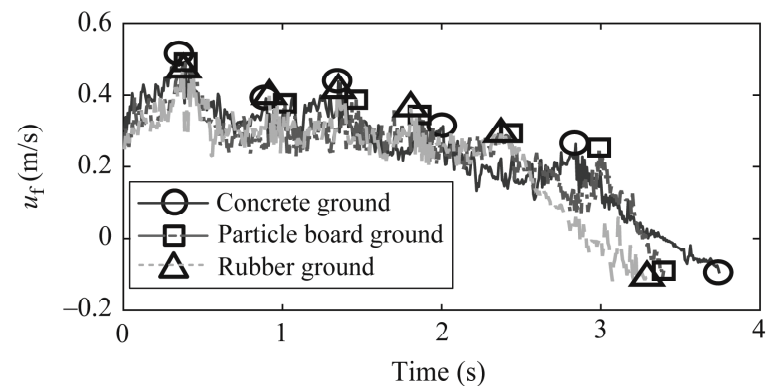

Figure 10. Transitioning: $u_{\mathrm{f}}$ versus time without AFC.

tracking of the virtual constraints. The following observations were made: (1) the desired virtual constraint values were approximate accurately applied to the robot; however, they lagged slightly owing to the limitation of the bus servo unit's tracking performance. (2) The initial state in each walking cycle was collected for online gait planning. Therefore, the accumulated error of each joint in previous cycles could be eliminated effectively.

Figure 10 shows the transition of the horizontal velocity of the biped's CoM $\left(u_{\mathrm{f}}\right)$ at the terminal time of the SSP on the three types of ground surfaces. The following results were observed: (1) during each walking cycle, $u_{\mathrm{f}}$ decreased during the first half of the cycle and increased in the second half; and (2) on the three types of ground surfaces, $u_{\mathrm{f}}$ decreased as the number of walking cycles increased. After the completion of five, six, and five walking cycles on the concrete, particle board, and rubber ground surfaces, respectively, the robot's walking speed did not satisfy the stability criterion and it fell down.

\subsection{Walking with AFC}

\subsubsection{Availability Verification of $A F C$}

To validate the effectiveness of the control strategy, AFC was added for walking experiments. Figure 11 illustrates 


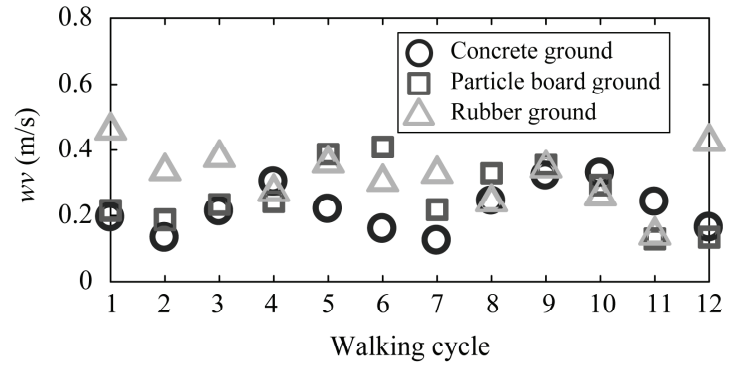

Figure 11. Transitioning: $w v$ versus walking cycle with AFC.

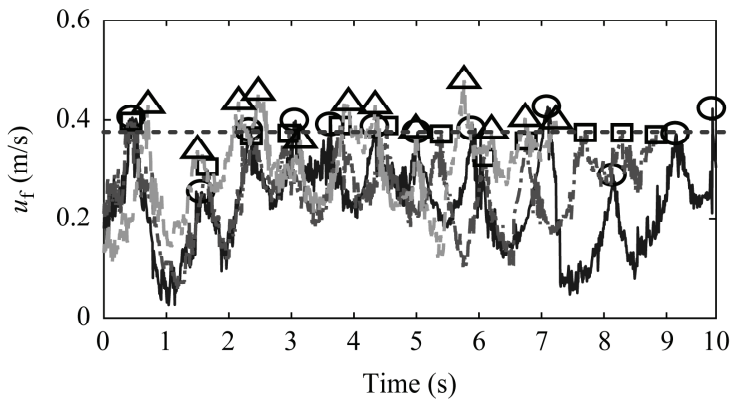

$\vartheta$ Concrete ground $\quad \square$ Particle board ground $-\Delta$ - Rubber ground

Figure 12. Transitioning: $u_{\mathrm{f}}$ versus time with AFC.

the transition of the average walking speed. Underactuated bipedal walking was achieved on the three types of ground surfaces with AFC, and the number of walking cycles was increased significantly. After 12 walking cycles, the average walking speeds of the robot on the concrete, particle board, and rubber ground surfaces were 0.1649, 0.1362 , and $0.4254 \mathrm{~m} / \mathrm{s}$, respectively; these speeds were determined to meet the stability criterion.

Figure 12 presents the transition of $u_{\mathrm{f}}$ at the terminal time of the SSP in the first 12 walking cycles on the three types of ground surfaces. The following results were observed: (1) $u_{\mathrm{f}}$ always fluctuated near $u_{\mathrm{f} 1}$ on the three types of ground surfaces with AFC; this is in accordance with the design objective of the control strategy. Consider, for example, the concrete ground surface; for the first walking cycle was $0.3829 \mathrm{~m} / \mathrm{s}$, and $u_{\mathrm{f}}$ for $3-12$ walking cycles fluctuated around this value with AFC. (2) The particle board surface had the highest $u_{\mathrm{f}}$, followed by the rubber and concrete surfaces. The times taken to complete 12 walking cycles on the concrete, particle board, and rubber surfaces were $7.245,8.834$, and $9.793 \mathrm{~s}$, respectively. (3) The control of $u_{\mathrm{f}}$ is not accurate, it does not affect the stability of robot walking. The controller must control only at terminal moment of the SSP during robot walking. Therefore, to achieve stable walking, implementing strict trajectory tracking control for the whole walking cycle as in other algorithms is not necessary.

\subsubsection{Adaptivity Verification of AFC}

To verify the adaptability of AFC, AFC was used in the walking experiments on the rubber-particle board ground surface [15]. Figure 13 shows the transition of $w v$ in three

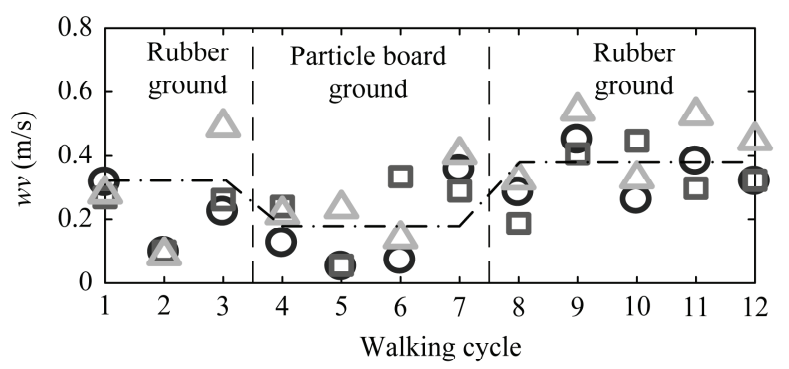

O First experiment $\square$ Second experiment $\triangle$ Third experiment

Figure 13. Transitioning: $w v$ versus walking cycle with AFC on rubber-particle board ground.

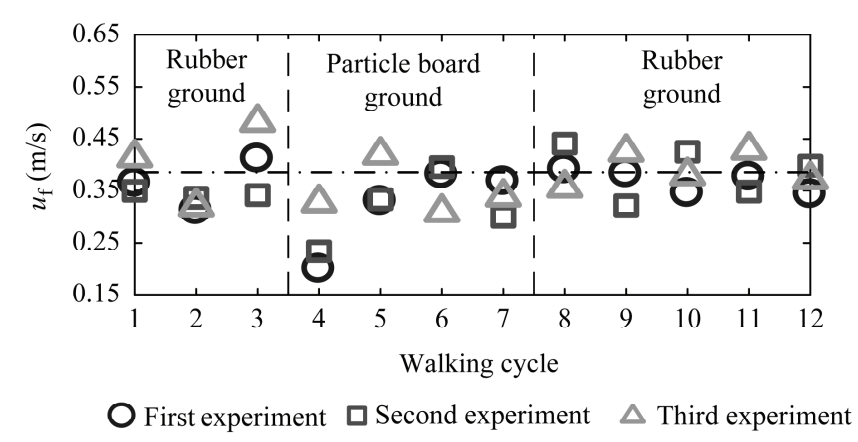

Figure 14. Transitioning: $u_{\mathrm{f}}$ versus walking cycle with AFC on rubber-particle board ground.

walking experiments on the rubber-particle board ground surface. Figure 14 presents the transition of $u_{\mathrm{f}}$ at the terminal time of the SSP. AFC was shown to adapt to different ground environments, and underactuated bipedal walking was achieved on the rubber-particle board ground. AFC was observed to always maintain the average walking speed within a certain range on different ground environments, and these speeds were determined to meet the stability criterion. The robot walking speed fluctuated for different ground surfaces, and the speed changes significantly lagged behind the ground surface changes. In the first experiment, the average walking speeds on the three types of ground surfaces were $0.2357,0.2095$, and $0.2210 \mathrm{~m} / \mathrm{s}$, respectively. The walking speed of the robot on the particle board ground surface was lower than that on the rubber ground surface; however, the speed change lagged behind the material change, and the lag distance was approximately $0.3 \mathrm{~m}$. The same result was obtained in the other two experiments.

\section{Conclusion}

This paper demonstrates the availability and effectiveness of an AFC method for underactuated bipedal walking on compliant ground with the real bipedal robot prototype UBbot. Dynamic walking with a step of $31 \%$ of the leg length was successfully realized on a ground surface made of three material types: concrete, particle board, and rubber.

(1) A more direct and simpler walking stability criterion is applied in the method. The robot will be stable 
if the walking speed can always be maintained in a range that is proved available for realizing at least one walking cycle.

(2) The walking system is simplified as an SISO system using a polynomial with a definite number of degrees. The algorithm controls the robot gait at the terminal moment of the SSP, and implementing strict trajectory tracking control for the whole walking cycle is not necessary.

\section{Acknowledgement}

This research is supported by National Natural Science Foundation of China (NSFC, Grant No.51675385 and No. $51175383)$.

\section{References}

[1] S. Collins, A. Ruina, R. Tedrake, and M. Wisse, Efficient bipedal robots based on passive-dynamic walkers, Science, 307(5712), 2005, 1082-1085.

[2] C. Chevallereau, G. Abba, Y. Aoustin, F. Plestan, E. Westervelt, C.C. De Wit, and J. Grizzle, Rabbit: A testbed for advanced control theory, IEEE Control Systems Magazine, 23(5), 2003, 57-79.

[3] K. Sreenath, H.W. Park, I. Poulakakis, and J.W. Grizzle, A compliant hybrid zero dynamics controller for stable, efficient and fast bipedal walking on MABEL, International Journal of Robotics Research, 30(9), 2011, 1170-1193.

[4] H.H. Zhao, W.L. Ma, M.B. Zeagler, and A.D. Ames, Humaninspired multi-contact locomotion with AMBER2, ACM/IEEE International Conference on Cyber-Physical Systems, Berlin, Germany, 2014, 199-210.

[5] T. Wang, Stable walking control of a 3D biped robot with foot rotation, Robotica, 32(4), 2014, 551-570.

[6] T. Wang, C. Chevallereau, and C.F. Rengifo, Walking and steering control for a 3D biped robot considering ground contact and stability, Robotics \& Autonomous Systems, 60(7), 2012, 962-977.

[7] B. Tondu and N. Bardou, A new interpretation of Mori's uncanny valley for future humanoid robots, International Journal of Robotics and Automation, 26(3), 2011, 1.

[8] S.H. Hyon, Compliant terrain adaptation for biped humanoids without measuring ground surface and contact forces, IEEE Transactions on Robotics, 25(1), 2009, 171-178.

[9] E.R. Westervelt, B. Morris, and K.D. Farrell, Analysis results and tools for the control of planar bipedal gaits using hybrid zero dynamics, Autonomous Robots, $23(2)$, 2007, 131-145.

[10] J. Aguilar and D.I. Goldman, Robophysical study of jumping dynamics on granular media, Nature Physics, 12(3), 2016, $278-283$.

[11] E.R. Westervelt, G. Buche, and J.W. Grizzle, Experimental validation of a framework for the design of controllers that induce stable walking in planar bipeds, International Journal of Robotics Research, 23(6), 2004, 559-582.

[12] P. Manoonpong, T. Geng, B. Porr, and F. Worgotter, The RunBot architecture for adaptive, fast, dynamic walking, IEEE International Symposium on Circuits and Systems, New Orleans, USA, 2007, 1181-1184.

[13] Y. Wang, J. Ding, and X. Xiao, An adaptive feedforward control method for underactuated bipedal walking on the compliant ground, International Journal of Robotics and Automation, 32(1), 2017, 63-77.

[14] J. Ding, X. Xiao, and Y. Wang, Preview control with adaptive fuzzy strategy for online biped gait generation and walking control, International Journal of Robotics and Automation, $31(6), 2016,496-508$.

[15] https://v.youku.com/v_show/id_XMzYzMTU1OTk0NA==. $\mathrm{html} ? \mathrm{spm}=\mathrm{a} 2 \mathrm{~h} 0 \mathrm{k} \cdot 8191407.0 .0 \&$ from $=\mathrm{s} 1.8-1-1.2$

\section{Biographies}

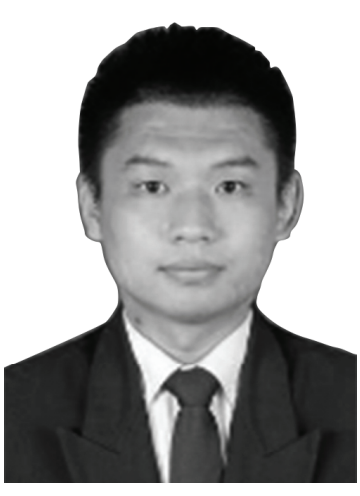

Daojin Yao obtained his B.S. in Mechanical and Electronic Engineering from the East China Jiaotong University, Nanchang, in 2012. He obtained his M.S. in Mechanical and Electronic Engineering from the Wuhan University of Technology, Wuhan, in 2015. Currently, he is pursuing a Ph.D. in Mechanical and Electronic Engineering from the Wuhan University, Wuhan, China. His research interests include robot control and embedded programming.

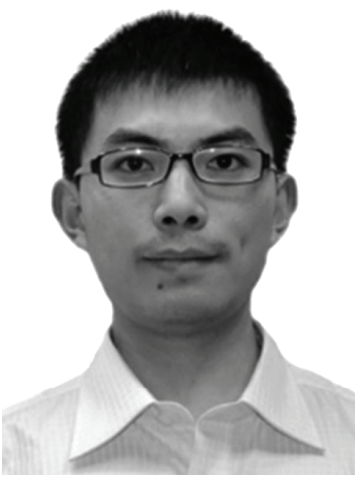

Yao Wu obtained his B.S. in Communication Engineering from the Beijing Information Science and Technology University, Beijing, in 2012. He obtained his M.S. in Machine Manufacture and Automation from the Beijing Technology and Business University, Beijing, in 2015. Currently, he is pursuing the Ph.D. degree in Mechanical Engineering from the Wuhan University, Wuhan, China. His research interests include biped robot dynamics and control.

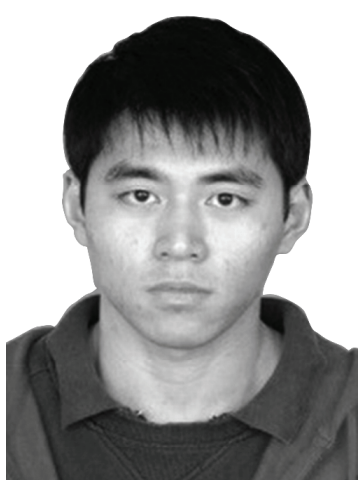

Yang Wang obtained his B.S. in Mechanical Design, Manufactory and Automation from Hubei University of Technology, Wuhan, in 2009. He obtained his Ph.D. degree in Mechanical Engineering from the Wuhan University, Wuhan, in 2017. His research interests include dynamics, planning, and control of biped robot walking in flexible environment.

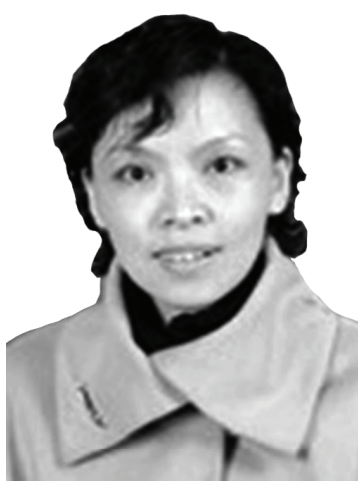

Xiaohui Xiao received the B.S. and M.S. degrees in Mechanical Engineering from the Wuhan University, Wuhan, China, in 1991 and 1998, respectively, and the Ph.D. degree in Mechanical Engineering from the Huazhong University of Science and Technology, Wuhan, China, in 2005. She is currently a Full Professor with the Mechanical Engineering Department, School of Power and

Mechanical Engineering. Her current research interests include mobile robotics, high-precision positioning control, and signal processing. 